\title{
Mögliche Therapiestrategien bei Covid-19-Erkrankungen mit chinesischen Arzneimitteln
}

\author{
Christian Thede, Neustadt/Holstein
}

Zusammenfassung: Angesichts der schweren CoronaVirus-Pandemie und aktuell noch nicht absehbarer verfügbarer kausaler Therapieansätze (Impfung, antivirale Medikamente) ist es von großer Bedeutung zu wissen, was die Chinesische Medizin zur Behandlung von Covid-19 beisteuern kann. Bereits während der SARS-Epidemie 2003, verursacht durch SARS-CoV-1, wurde nach einem 2004 veröffentlichten Bericht der WHO in China sowohl präventiv als auch therapeutisch zusätzlich zur westlichen Medizin mit Chinesischer Medizin behandelt. Dabei ließ sich sowohl präventiv als auch kurativ eine signifikante Wirksamkeit nachweisen. Während des aktuellen Ausbruchs von SARS-CoV-2 sind in der Region Wuhan allein bis Ende Februar 2020 ca. 60.000 Covid-19-Patienten mit Chinesischer Medizin behandelt worden. In dieser
Arbeit wird im ersten Teil ein zusammenfassender Überblick über eine Reihe von Quellen mit Behandlungsempfehlungen und Erfahrungen verschiedener Kliniken, Arbeitsgruppen und offizieller Stellen gegeben. Im zweiten Teil werden - basierend auf den aktuell bekannten Informationen - eigene Überlegungen zur Pathophysiologie und zu wichtigen Therapieprinzipien angestellt. Abschließend werden vorläufige Behandlungsvorschläge mit chinesischen Arzneimitteln für das offenbar zentrale Dysharmoniemuster - eine Blockade des qi pulmonale (Qi des Fk "Lunge", feiqi) und der Transformation von Flüssigkeiten im Rahmen einer Akkumulation von humor („,Feuchtigkeit", shi) mit Toxischem - für die sich bei schweren Verläufen entwickelnde Pneumonie vorgestellt.

Schlüsselwörter: Covid-19 · SARS-CoV-2 · Chinesische Phytotherapie · Pneumonie · Blockade des qi pulmonale (Qi des Fk "Lunge", feiqi) · Humor („Feuchtigkeit", shi) · Toxisches

Chin Med 2020;35:55-78

\section{Possible therapy strategies for Covid 19 infections using Chinese Herbs}

\begin{abstract}
In view of the severe corona virus pandemic and the not yet foreseeable availability of causal therapy approaches (vaccination, antiviral drugs), it is of great importance to know what Chinese medicine can contribute to the treatment of Covid 19. According to a WHO report published in 2004, concerning the 2003 SARS epidemic caused by SARSCoV-1, Chinese medicine was used in China both preventively and therapeutically in addition to Western medicine. In both these preventive and curative roles, treatment proved to be significantly effective. During the current outbreak of SARS-CoV-2, about 60,000 Covid 19 patients were treated with
\end{abstract}

Chinese medicine in the Wuhan region alone by the end of February 2020.

The first part of this paper provides a summarizing overview of a number of sources with treatment recommendations and experiences of different clinics, working groups and official bodies. In the second part - based on currently known information - the author voices his own considerations on pathophysiology and important therapeutic principles. Finally, he presents preliminary treatment proposals using Chinese medicinal remedies for what appear to be the central pattern of the disorder - a blockage of pulmonary qi and the transformation of fluids in the context of an accumulation of dampness /humor with toxicity and, in severe cases, development into pneumonia.

Key Words: Covid-19·SARS-CoV-2 · Chinese phytotherapy $\cdot$ Pneumonia · Blockage of pulmonary qi · Accumulation of dampness/ humor · Toxicity 
Typische Verläufe

\section{Einleitung}

1.1 Kurzinformationen zu den Charakteristika von SARS-CoV-2 und der Covid-19-Erkrankung

SARS-CoV-2 ist durch eine extrem hohe Infektiosität gekennzeichnet, während die Letalität nach aktuellen Informationen (Stand März 2020) mit vermutlich unter $1 \%$ leicht, aber nicht wesentlich höher als die der Influenza einzuschätzen ist. Der Großteil der Infektionen verläuft blande oder nur mit milden Symptomen, wobei zum einen auch asymptomatische Infizierte infektiös sein können und zum anderen die Phase der Infektiösität bei Personen, die erkranken, bereits deutlich vor Ausbruch der Symptome beginnt. Initiale Symptome eines Infekts der oberen Atemwege sind oft nur mild ausgeprägt (1. Erkrankungswoche). Infolgedessen wird die Erkrankung oft erst klinisch auffällig, wenn erste Zeichen der sich entwickelnden interstitiellen Pneumonie erkennbar werden (2. Woche). Das Risiko für einen schweren Verlauf - und somit auch die Letalität der Erkrankung - nimmt mit zunehmendem Lebensalter zu und ist bei einer Reihe von schwächenden Vorerkrankungen erhöht. Die Pneumonie erstreckt sich als atypische Pneumonie oft netzförmig auf alle Lungenabschnitte und kann bei schweren Verläufen in eine überschießende Immunreaktion münden (3. Woche), gekennzeichnet durch einen sogenannten „Zytokinsturm“, der zu einer interstitiellen und alveolären Überladung mit Immunzellen, Entzündungsmediatoren und Flüssigkeit führt, was letztlich in die respiratorische Insuffizienz führt und eine prolongierte Beatmung mit sehr hohem endexpiratorischem Druck (PEEP) unumgänglich macht. Diese Faktoren führen offenbar - teilweise aufgrund von Umbauprozessen, jedoch auch durch Schwächung der Atemmuskulatur zunächst zu einer schwierigen Entwöhnung von der maschinellen Beatmung sowie nach Überstehen der Akuterkrankung zu fortbestehenden restriktiven Ventilationsstörungen.

\subsection{Bisherige Erfahrungs- und Behandlungsberichte aus China über die Behandlung von Covid-19- Erkrankten mit Chinesischer Medizin und Quellenübersicht}

Bereits während der SARS-Epidemie 2003, verursacht durch SARS-CoV-1, wurden in China Patienten zusätzlich zur westlichen Medizin mit Chinesischer Medizin behandelt. In einem 2004 veröffentlichten Bericht der WHO [1] wurde im Rahmen einer kontrollierten Beobachtungsstudie eine signifikant höhere Überlebensrate bei Patienten festgestellt, die eine kombinierte Behandlung aus westlicher und Chinesischer Medizin erhielten, verglichen mit einer Gruppe, die ausschließlich mit westlicher Medizin behandelt wurde.

Während des aktuellen Ausbruchs von SARS-CoV-2 sind in der Region Wuhan allein bis Ende Februar 2020 ca. 60.000 Covid-19-Patienten mit Chinesischer Medizin behandelt worden [2]. Erste Erfahrungs- und Behandlungsberichte darüber liegen nun in westlichen Sprachen vor. Die Berichte, auf die in der vorliegenden Arbeit Bezug genommen werden wird, sind von verschiedenen Seiten zur Verfügung gestellt worden. Heiner Fruehauf (Gründer des College of Classical Chinese Medicine in Portland, USA, renommierter Autor vieler Artikel zur Chinesischen Medizin), John K. Chen (Mitautor von Chinese Medical Herbology and Pharmacology), Axel Wiebrecht vom CTCA (Centrum für Therapiesicherheit in der Chinesischen Arzneimitteltherapie) und Nina Zhao-Seiler für ihre Übersetzungsarbeit gilt dafür unser aller Dank. 
Folgende Quellen sind für diese Arbeit gesichtet und ausgewertet worden:

$\rightarrow$ Übersetzter Auszug aus: „Diagnose- und Behandlungsplan bezüglich der durch das neuartige Coronavirus verursachten Pneumonie (7. provisorische Auflage)“, übersetzt von Nina Zhao-Seiler [3]

$\rightarrow$ „Medical Records from a Young and Brave Female Traditional Chinese Medicine (TCM) Doctor on Fighting the COVID-19“', Chen Juan et al. [4]

$\rightarrow$ „Initial Thoughts on Coronavirus Prevention and Treatment with Chinese Medicine", Ausschnitt aus einem Artikel von Heiner Fruehauf [5]

$\rightarrow$ „Report from the Front Line in Wuhan“, Liu Lihong, übersetzt von Heiner Fruehauf [6]

$\rightarrow$ „The Dampness Epidemic: Exploring the Clinical Characteristics of COVID-19 in Shanghai", Xue Yan et al. [7]

$\rightarrow$ Therapieprotokolle folgender Kliniken: Hubei Provincial Hospital of TCM, Wuhan Union Hospital; in: „How Coronavirus (COVID-19) is treated with TCM in China“, John Chen [2]

Die Inhalte in den hier genannten Quellen über Behandlungsberichte bzw. Therapieprotokolle werden in diesem Artikel zusammengefasst und kommentiert. Für eine vollständigere Einsicht wird auf das „Open Access Paper“ [8] auf der SMS-Homepage und das Quellenverzeichnis verwiesen.

\section{Zusammenfassung der Therapiestrategien aus verschiedenen Quellen mit Kommentaren}

2.1 Diagnose- und Behandlungsplan bezüglich der durch das neuartige Coronavirus verursachten Pneumonie (7. provisorische Auflage) [3]

In diesem Therapieprotokoll werden Therapieempfehlungen für folgende Phasen der Covid-19-Erkrankung angegeben:

\subsubsection{Medizinische Beobachtungsphase}

Für den Fall, dass als führende Symptomatik Kraftlosigkeit und Verdauungsbeschwerden bestehen, wird die „Kapsel mit Agastache zur Stärkung des geradläufigen Qi“ (Huoxiang zhengqi jiaonang) empfohlen, also eine Rezeptur mit extima (Oberfläche, biao) befreiender Wirkung, die zugleich die „Mitte“ harmonisiert und humor („Feuchtigkeit“, shi) umwandelt. Damit wird bereits dem Umstand Rechnung getragen, dass sich im Fall eines schweren Verlaufs in der Regel eine Akkumulation von humor („Feuchtigkeit“, shi) ober- und unterhalb des Zwerchfells entwickelt.

Das hier für Kraftlosigkeit und Fieber angegebene „Pulver mit Lonicera und Forsythia“ (Yinqiao san) behandelt eine calor-venti-Affektion („Wind-Hitze“, fengre) und eignet sich auch für die bei einer SARS-CoV-2-Infektion häufigen Initialsymptome wie Husten und Halsschmerzen.

\subsubsection{Gesamte klinische Behandlungsphase}

Hier wird eine Rezeptur angegeben, die offenbar für den Einsatz bei extremem Zeitmangel bei vielen Patienten konzipiert wurde. Die „Den o. pulmonalis (Fk ,Lunge') kühlendes und Toxisches ausscheidendes Dekokt“" (Qingfei paidu tang) genannte Rezeptur kann als „Universalspezifikum“ für sämtliche Stadien und Schweregrade bezeichnet werden und beinhaltet neben den Hauptbestandteilen von „Kleines Bupleurum-Dekokt“ (Xiao chaihu tang) vorwiegend Arzneien zur Umwandlung und Ausleitung von humor („Feuchtigkeit“, shi), zur Stützung und

Empfohlene Rezeptur bei Kraftlosigkeit und Verdauungsbeschwerden 
Blockiertes qi pulmonale (Qi des Fk „Lunge“, feiqi)

Epidemisch-Toxisches (yidu)
Harmonisierung der „Mitte“, zur Regulation des qi pulmonale (Qi des Fk „Lunge“, feiqi) bzw. zur Hustenstillung sowie zur Ausleitung von persistierenden exogenen pathogenen Agenzien über die extima (Oberfläche, biao).

\subsubsection{Leichte Verläufe}

In diesem Abschnitt wird zunächst ein Konzept für „Einstauung von humor algidus (,Kälte-Feuchtigkeit') im o. pulmonalis (Fk ,Lunge')“ (hanshi yu fei 寒湿郁肺) empfohlen, das mit sich ergänzenden Modulen das offenbar zentrale Dysharmoniemuster schwerer Verläufe von Covid-19 in den Fokus nimmt, nämlich die Umwandlung und Kanalisierung von trübem humor („Feuchtigkeit“, shi). Die Hauptarzneien aus „Dekokt mit Ephedra, Prunus armeniaca, Gypsum und Glycyrrhiza“ (Maxing shigan tang) mit moderater Dosierung von Gypsum fibrosum (Shigao) dienen hier vor allem der Regulierung des blockierten qi pulmonale (Qi des Fk „Lunge“, feiqi), mehrere weitere Arzneien bewirken die Umwandlung und Kanalisierung von humor („Feuchtigkeit“, shi), unterstützt durch die Harmonisierung der „Mitte“ durch Qi regulierende und verdauungsfördernde Ingredienzien.

Ein weiteres Konzept für leichte Verläufe ist die Therapie von „Einlagerung von calor humidus (,Feuchtigkeit-Hitze) im o. pulmonalis (Fk ,Lunge)" (shire yun fei 湿热蕴肺). Bei der angegebenen Rezeptur fällt auf, dass auch bei einer humorProblematik („Feuchtigkeit“, shi) mit angenommenem calor-Aspekt („Hitze“, re) darauf geachtet wird, mit aromatischen Arzneien warmen Temperaturverhaltens eine Umwandlung des Trüben sicherzustellen, was die Annahme unterstützt, dass die Therapie der Blockade des qi pulmonale (Qi des Fk „Lunge“, feiqi) und die Elimination einer Akkumulation von humor („Feuchtigkeit“, shi) von zentraler Bedeutung ist.

\subsection{4 Übliche Verläufe}

Wie im vorherigen Abschnitt wird auch in diesem jeweils ein Konzept für eine Akkumulation von humor („Feuchtigkeit“, shi) ohne bzw. mit calor-Aspekt („Hitze“, re) vorgestellt, und dafür werden Rezepturen mit etwas von denen im vorhergehenden Abschnitt abweichender Zusammensetzung angegeben. Wie in den meisten anderen Rezepturen aus diesem Therapieprotokoll findet sich auch hier Ephedrae herba (Mahuang) in Kombination mit aromatischen Arzneien sowohl zur Elimination der Akkumulation von humor („Feuchtigkeit“, shi) als auch der Entfaltung des qi pulmonale (Qi des Fk „Lunge“, feiqi).

\subsubsection{Schwere Verläufe}

Mit dem „Blockade des o. pulmonalis (Fk,Lunge') durch Epidemisch-Toxisches“ (yidu bifei 疫毒闭肺) genannten Dysharmoniemuster wird bereits ein Schweregrad der Covid-19-Erkrankung beschrieben, der Therapeuten der Chinesischen Medizin allenfalls in seltenen Ausnahmefällen zugänglich sein dürfte. Obwohl das Krankheitsmuster „yidu“ (Epidemisch- Toxisches) genannt wird, wird auch hier primär die Kombination von „Dekokt mit Ephedra, Prunus armeniaca, Gypsum und Glycyrrhiza" (Maxing shigan tang) mit aromatischen und diuretischen Arzneien zur Lösung der humor-Akkumulation („Feuchtigkeit“, shi) und somit der Blockade des qi pulmonale (Qi des Fk „Lunge“, feiqi) eingesetzt. Rhei rhizoma (Dahuang), Lepidii/Descurainiae semen (Tinglizi) und Paeoniae radix rubra (Chishaoyao) dienen der Ableitung des calor-Aspekts („Hitze“, re).

Das für ein „Emporlodern des Qi und des qi constructivum (Bauenergie)“ ( $q i$ ying liang fan 气营两燔) genanntes Muster empfohlene Rezept ist letztlich eine 
Modifikation von „Dekokt zur Kühlung der Bauenergie“ (Qingying tang), ergänzt durch Gypsum fibrosum (Shigao) und Anemarrhenae rhizoma (Zhimu), also zur Klärung von calor („Hitze“, re) vorwiegend in der Schicht des qi constructivum (Bauenergie, yingqi), aber auch der Qi-Schicht nach der Theorie der morbi temperati („Wärme“-Erkrankungen, wenbing). Es geht hier also um sehr schwer Erkrankte, die meist bewusstlos, eventuell beatmungspflichtig sind und bei uns in aller Regel nicht mit Chinesischer Medizin behandelt werden können bzw. dürfen.

\subsubsection{Schwere Verläufe in kritischem Zustand}

Die in diesem Abschnitt angegebene Variante von „Dekokt mit Ginseng und Aconitum “(Shenfu tang) ist bei einem extremen Defizit des qi originale (UrsprungsQi, yuanqi) bei gleichzeitigem Kollaps des Yang indiziert - einem drohenden bzw. aktuellen Herz-Kreislauf-Versagen entsprechend - und wird in China in derartigen Fällen parenteral verabreicht, was bei uns nicht realisierbar ist.

\subsubsection{Erholungsphase}

Für diese Phase wird bei einer „depletio (energetische Schwäche, $x u$ ) der oo. pulmonalis et lienalis (Fk ,Lunge' und ,Milz')“(fei pi qixu 肺脾气虚) eine Modifikation von „Dekokt der sechs Edlen“ (Liu junzi tang) angegeben.

Weiterhin wird eine Rezeptur für den Fall eines Defizits des Yin mit calor depletionis („Hitze“ aufgrund energetischer Schwäche, xure) angegeben, mit Zutaten zur Ergänzung des Yin, zur Kühlung und Befeuchtung.

\subsubsection{Zusammenfassung und Kommentar}

Es wird in dieser Sammlung von Therapieprotokollen die Entwicklung einer Akkumulation von humor („Feuchtigkeit“, shi) und die daraus resultierende Blockade des qi pulmonale (Qi des Fk „Lunge“, feiqi) als zentrale Problematik verschiedener Stadiendifferenzierungen angesehen und therapeutisch adressiert. Abgesehen von den Musterdifferenzierungen, bei denen calor-Aspekte („Hitze“, re) absolut dominant sind, werden als zentrale Arzneien immer wieder aromatische Arzneien und Ephedrae herba (Mahuang) bzw. das „Dekokt mit Ephedra, Prunus armeniaca, Gypsum und Glycyrrhiza" (Maxing shigan tang) angegeben, um die Akkumulation im o. pulmonalis (Fk „Lunge“, fei) zu lösen und die Umwandlung und Kanalisierung von humor („Feuchtigkeit“, shi) zu gewährleisten.

\subsection{Erfahrungsbericht über Behandlungen von Patienten mit Covid-19-Erkrankung in China [4]}

\subsubsection{Behandlungs- und Befundbericht einer chinesischen Arbeitsgruppe} um die Ärztin Chen Juan über 30 klinisch stationäre Covid-19-Patienten

Chen Juan berichtet über 30 Patienten mit folgender Hauptsymptomatik: Fieber, sehr häufig trockener Husten mit wenig oder keinem Auswurf, teilweise mit Kälteabneigung, häufig kalten Extremitäten und mit folgendem typischen Zungenbefund: meist blasse, gedunsene Zungen mit dicken, weißen, schmierigen Belägen. Die dazugehörigen Zungenfotos sind in dieser Veröffentlichung enthalten. Weiter wird von regelmäßig erschwerter Inspiration bei normaler Exspiration berichtet und dass erfolgreiches Abhusten von Schleim die Atmung bedeutend erleichtere. Die Gruppe ging von folgender Hauptdiagnose in der Anfangsphase der Erkrankung aus: humor algidus („Kälte-Feuchtigkeit“, hanshi) dringt in den o. pulmonalis (Fk „Lunge“, fei) ein. Im Rahmen eines integrierten
Behandlung in der Erholungsphase

Therapie von 30 stationären Patienten 
Die Wirkung der wichtigen Arznei Ephedrae herba (Mahuang)
Ansatzes mit westlicher und Chinesischer Medizin wurden zunächst Konzepte auf der Basis folgender Rezepturen eingesetzt: „Kleines Bupleurum-Dekokt“ (Xiao chaihu tang), „Dekokt mit Bupleurum und Zimtzweigen“ (Chaihu guizhi tang), „Dekokt mit Ephedra, Aconitum und Asarum“ (Mahuang fuzi xixin tang) und „Dekokt mit Trichosanthis fructus, Allii bakeri bulbus und Pinelliae rhizoma“ (Gualou xiebai banxia tang).

Sowohl diese als auch andere Arbeitsgruppen haben offenbar in der Phase des Massenanfalls von Erkrankten in Wuhan eine Kombination der vier genannten Rezepturen als Patentmittel für Covid-19-Patienten in verschiedenen Erkrankungsstadien ausgegeben.

Chen Juan berichtet über verkürzte Phasen hohen Fiebers bei Verzicht auf Antipyretika und interpretiert die dort teilweise beobachteten Leberfunktionsstörungen als Folgen der Gabe antiviraler Medikamente. Sie äußert die Auffassung, dass Interventionen, die das qi orthopathicum (geradläufiges $\mathrm{Qi}$, zhengqi) beeinträchtigen, wie antivirale und antipyretische Medikamente, aber auch ein Übermaß an kühlenden chinesischen Arzneimitteln, den Flüssigkeitsmetabolismus zu sehr schädigen könnten und somit vermieden werden sollten.

Die Autorin betont die Wichtigkeit des Einsatzes von pituita („Schleim“, tan) eliminierenden Arzneien im weiteren Verlauf der Erkrankung. Des Weiteren hebt sie die essentielle Bedeutung der Verwendung von Ephedrae herba (Mahuang) in nahezu allen Krankheitsstadien hervor und begründet diese Auffassung damit, dass im Fall von Covid-19-Erkrankungen bei der Anwendung von Ephedrae herba (Mahuang) nicht die extima (Oberfläche, biao) freimachende und Schwitzen auslösende Wirkung im Vordergrund steht, sondern die das qi pulmonale (Qi des $\mathrm{Fk}$ „Lunge“, feiqi) lösenden und die Wasserwege freimachenden und diuretischen Wirkkomponenten der Arznei, die somit zur Zerteilung und Kanalisierung der Akkumulation von humor pituitae („Schleim-Feuchtigkeit“, tanshi) im o. pulmonalis (Fk „Lunge“, fei) beitragen.

In der Prävention hält Chen Juan in vielen Fällen den Einsatz von Aconiti radix lateralis praeparata (Fuzi), etwa im Kontext der Rezeptur „Die Mitte regulierende Aconit-Pille“ (Fuzi lizhong wan), für besonders bedeutsam, mit der Begründung, dass das abgelaufene Jahr aus der Perspektive der Phasenenergetik ungünstig für die Energien der Wandlungsphase Erde gewesen sei und somit bei vielen Menschen die „Mitte“ gestärkt und erwärmt werden solle.

Nach Angaben der Autorin konnten alle 30 Patienten, über die hier berichtet wird, gesund bzw. gebessert entlassen werden, wobei eingeräumt wird, dass lediglich vier Patienten höheren Alters und schwer erkrankt waren.

\subsubsection{Zusammenfassung und Kommentar}

In der vorliegenden kleinen Beobachtungsstudie wurden Patienten unter der Hauptdiagnose „humor algidus (,Kälte-Feuchtigkeit', hanshi) dringt in den o. pulmonalis ( $\mathrm{Fk}$,Lunge', fei) ein und führt zur Akkumulation von humor (,Feuchtigkeit, shi) und pituita (,Schleim, tan) mit Blockade des qi pulmonale (Qi des Fk ,Lunge, feiqi)" behandelt. Die Therapie zielte auf Umwandlung und Kanalisierung der Akkumulation der pathologischen Flüssigkeiten und auf die Lösung des $q i$ pulmonale (Qi des Fk „Lunge“, feiqi) unter besonderer Berücksichtigung von Ephedrae herba (Mahuang) ab. Alle Patienten im beobachteten Kollektiv konnten die Klinik offenbar gesundet bzw. gebessert verlassen. 


\subsection{Initial Thoughts on Coronavirus Prevention and Treatment with Chinese Medicine [5]}

Der hier wiedergegebene Erfahrungsbericht bestätigt die in den „staatlichen“ chinesischen Texten und in dem von John Chen weitergegebenen Erfahrungsbericht vertretene Auffassung, dass die zentrale Pathologie der fortgeschrittenen und schweren Covid-19-Erkrankung die Akkumulation von humor („Feuchtigkeit", shi) ist und deren Auflösung und Kanalisierung das primäre Therapieprinzip sein sollte, um den Fluss des qi pulmonale (Qi des Fk „Lunge“, feiqi) wiederherzustellen. Auch hier wird die Entwicklung von calor-Symptomen („Hitze“, re) als sekundärer Prozess angesehen.

\subsection{Report from the Front Line in Wuhan [6]}

\subsection{1}

Der vollständige Text beinhaltet die Angabe, dass 10 stationäre Patienten behandelt wurden, und berichtet über regelmäßig beobachtete Zeichen und Symptome wie dicke, weiße, schmierige Zungenbeläge, pp. lubrici („schlüpfrige“ Pulse, maihua) an der rechten cun-Position und unproduktiver Husten. In theoretischen Überlegungen mit Bezug auf die „Abhandlung über schädigende Kälte (algor laedens)“ (Shanghan lun, von Zhang Zhongjing, aus dem 2. Jh.) wird die Auffassung vertreten, dass in der Therapie der komplexe Charakter der Covid-19-Erkrankung berücksichtigt werden sollte. Als Beispiel für ein Konzept, das sowohl den yangmaior-Anteil (Großes Yang, taiyang) als auch den des splendor yang (Überstrahlung des Yang, yangming) behandelt, wird das „Dekokt mit Ephedra, Prunus armeniaca, Gypsum und Glycyrrhiza“ (Maxing shigan tang) vorgeschlagen.

Der bei vielen Patienten unproduktive Husten wird hier als ein Zeichen zäher, festsitzender und "trockener“ pituita („trockener Schleim“, zaotan) interpretiert, der nicht allein durch Umwandlung, sondern durch Befeuchten und Erweichen gelöst werden soll, wofür in diesem Text besonders folgende Arzneimittel empfohlen werden: Benincasae semen (Dongguazi), Trichosanthis semen (Gualouren), Raphani semen (Laifuzi), Sinapis albae semen (Baijiezi).

Empfehlungen zu vollständigen Rezepturen werden in diesem Text nicht angegeben. Über Erfolg oder Misserfolg der Behandlungen werden keine konkreten Angaben gemacht.

\subsubsection{Zusammenfassung und Kommentar}

Der Autor Liu Lihong vertritt grundsätzlich die Auffassung, dass bei der Therapie von Covid-19-Erkrankten der Komplexität des Krankheitsgeschehens Rechnung getragen werden muss. Dabei wird besonders auf Differenzierungsmodelle nach der „Abhandlung über schädigende Kälte (algor laedens)“ (Shanghan lun, s.o.) Bezug genommen. Außerdem wird die Bedeutung von humor („Feuchtigkeit“, shi) und "trockener“ pituita („trockener Schleim“, zaotan) bei Diagnose und Therapie hervorgehoben.

\subsection{The Dampness Epidemic: Exploring the Clinical Characteristics of COVID-19 in Shanghai [7]}

In diesem Artikel wird die Bedeutung von „Einstauung von humor-Toxischem (,Feuchtigkeit') im o. pulmonalis (Fk,Lunge')“( shidu yufei 湿毒郁肺) als zentraler pathogenetischer Prozess hervorgehoben und unter Zuhilfenahme diverser klassischer Literaturstellen, darunter dem „Inneren Klassiker des Gelben Fürsten“
Akkumulation von humor („Feuchtigkeit", shi) bei schweren Verläufen

Differenzierung nach der „Abhandlung über schädigende Kälte

(algor laedens)“(Shanghan lun) 
Studien zur Prävention

Rezepturen für verschiedene

Pneumonie-Formen
(Huangdi Neijing, aus dem 1. Jh. v.u.Z., im 8. und 11. Jh. überarbeitet) ausführlich begründet. Es wird beschrieben, wie die Akkumulation von humor („Feuchtigkeit“, shi) bzw. calor humidus („Feuchtigkeit-Hitze“, shire) den o. pulmonalis (Fk „Lunge“, fei) schädigt und blockiert und zunehmende Stagnation zur Entwicklung von calor („Hitze“, re) und Toxischem $(d u)$ führt, schließlich zu einer Affektion und gravierenden Schädigungen der Ebenen des Xue und des qi constructivum (Bauenergie, yingqi). Konkrete therapeutische Empfehlungen oder Erfahrungen werden hier nicht genannt.

2.6 Covid-19-Therapieprotokoll des Hubei Provincial Hospital [2]

In diesem Text werden Behandlungsempfehlungen für verschiedene Phasen und Schweregrade der Erkrankung angegeben, die im Folgenden zusammengefasst und erläutert werden.

\subsubsection{Prävention}

Zur Prävention der Erkrankung wird eine Modifikation von „Pulver gegen Wind aus Jade" (Yu pingfeng san) mit folgenden Ergänzungen empfohlen: Dryopteridis rhizoma (Guanzhong), Lonicerae flos (Jinyinhua), Citri reticulatae pericarpium (Chenpi), Eupatorii herba (Peilan).

Während des zurückliegenden SARS-1-Ausbruchs haben in China Mitarbeiter medizinischer Einrichtungen an einigen Studien teilgenommen, in denen das „Pulver gegen Wind aus Jade“ (Yu pingfeng san) mit Ergänzungen zur Prävention eingenommen wurde. Keiner der insgesamt über 4.500 Mitarbeiter der VerumGruppen soll infiziert worden sein. Angesichts der extrem hohen Infektiösität von SARS-CoV-2 dürfte eine Infektion bei Exposition kaum zu vermeiden sein. Der präventive Effekt bezieht sich darauf, dem Fortschreiten der Covid-19-Erkrankung von der extima (Oberfläche, biao) in die intima (Inneres, li) entgegenzuwirken.

\subsubsection{Frühphase}

Für diese Phase der Erkrankung werden Konzepte für drei verschiedene Dysharmoniemuster angegeben, die hier verkürzt wiedergegeben werden:

$\rightarrow$ Algor venti („Wind-Kälte“, fenghan) affiziert die extima (Oberfläche, biao): „Pueraria-Dekokt“" (Gegen tang)

$\rightarrow$ Toxischer calor („Hitze“, re) attackiert den o. pulmonalis (Fk „Lunge“, fei): Modifikation von „Pulver mit Lonicera und Forsythia“ (Yinqiao san)

$\rightarrow$ Humor algidus („Kälte-Feuchtigkeit“, hanshi) im o. pulmonalis (Fk „Lunge“, fei): Für diese Situation wird eine Zusammenstellung aus scharfen und aromatischen Arzneimitteln angegeben, um algor („Kälte“, han) zu zerstreuen und humor („Feuchtigkeit“, shi) umzuwandeln. Ephedrae herba (Mahuang) dient zudem der Entfaltung des qi pulmonale (Qi des Fk „Lunge“, feiqi).

\subsubsection{Pneumonie-Stadien}

Zur Therapie dieser Stadien werden ebenfalls für verschiedene Muster differenzierte Konzepte angegeben:

$\rightarrow$ Yang-minor-Syndrom (Kleines Yang, shaoyang) mit humor („Feuchtigkeit“, shi): Hierfür wird eine modifizierte Kombination aus „Kleines Bupleurum-Dekokt“ (Xiao chaihu tang), „Dekokt der drei Samen“(Sanren tang) und „Gift auflösende Pille des süßen Taus" (Ganlu xiaodu dan) empfohlen. Mit dieser Kombination wird eine Harmonisierung des yang minor (Kleines Yang, shaoyang) sowie die 
aromatische Umwandlung von humor („Feuchtigkeit“, shi) und die Ausleitung von calor humidus („Feuchtigkeit-Hitze“, shire) angestrebt.

$\rightarrow$ Calor humidus („Feuchtigkeit-Hitze“, shire) affiziert den o. pulmonalis (Fk „Lunge“, fei): Für dieses Muster wird eine Kombination von „Dekokt mit Ephedra, Prunus armeniaca, Coicis und Glycyrrhiza“ (Maxing yigan tang) und „Kleines Dekokt gegen Vernichtungsgefühl in der Brust“ (Xiao xianxiong tang) empfohlen.

$\rightarrow$ „Blockade des o. pulmonalis (Fk ,Lunge) durch Epidemisch-Toxisches“ (yidu bifei 疫毒闭肺): Das hier angegebene Rezept basiert auf dem „Dekokt des weißen Tigers" (Baihu tang) zur Klärung eines gravierenden calor-Aspekts („Hitze“, re) in der Qi-Ebene (nach den morbi temperati, „Wärme“-Erkrankungen, wenbing), ergänzt durch Dioscoreae rhizoma (Shanyao) und Panacis quinquefolii radix (Xiyangshen) zur Unterstützung des Qi sowie Arzneien zur Lösung der im Rahmen des schweren Krankheitsverlaufs herrschenden Blockade.

$\rightarrow$ „Blockade im Inneren und Entweichen nach außen“ (neibi waituo 内闭外脱): Das angegebene Konzept zielt auf den drohenden Verlust des qi originale (Ursprungs-Qi, yuanqi) im Verein mit einem Kollaps des Yang ab, letztlich also ein potenziell präfinaler Zustand, der bei uns ausschließlich westlich-intensivmedizinisch behandelt wird. Es wird eine Kombination folgender Rezepturen angegeben: „Das Dekokt gegen die vier Kontravektionen mit Ginseng radix“ (Sini jia renshen tang), "Pille des befriedeten Palastes mit Bovis calculus“ (Angong niuhuang wan), „Pulver des purpurnen Schnees“(Zixue san).

\subsubsection{Erholungsphase}

Für einen Zustand nach Abklingen der Akutsymptomatik mit bereits gelinderter Atemnot wird eine Modifikation von "Glehnia- und Ophiopogon-Dekokt" (Shashen maidong tang) angegeben, um Yin und Säfte zu ergänzen und pituita („Schleim“, tan) zu lösen.

\subsubsection{Zusammenfassung und Kommentar}

Auch bei den Konzepten des Hubei Provincial Hospitals steht die Behandlung der Akkumulation von humor („Feuchtigkeit“, shi) im Zentrum der therapeutischen Anstrengungen, gefolgt von der Elimination der sich entwickelnden calor-Aspekte („Hitze“, re).

\subsection{Covid-19-Therapieprotokoll des Wuhan Union Hospital [2]}

In diesem Text werden Behandlungsempfehlungen für verschiedene Phasen und Schweregrade der Erkrankung angegeben, die im Folgenden zusammengefasst und erläutert werden.

\subsubsection{Prävention}

Um dem Fortschreiten der Covid-19-Erkrankung von der extima (Oberfläche, biao) in die intima (Inneres, $l i$ ) entgegenzuwirken, wird auch in dieser Quelle eine Modifikation von „Pulver gegen Wind aus Jade“ (Yu pingfeng san), ergänzt durch humor („Feuchtigkeit“, shi) umwandelnde und kanalisierende sowie desinfizierende Arzneien, empfohlen. Der Zusatz von Dryopteridis rhizoma (Guanzhong) erfolgt wie auch in anderen Rezepturen aus China wegen ihrer antiviralen Eigenschaften.
Behandlung nach Abklingen der Akutsymptomatik 
Eliminieren von humor („Feuchtigkeit", shi)
2.7.2 Calor venti („Wind-Hitze“, fengre) affiziert die extima (Oberfläche, biao) Zur Therapie wird hier eine Kombination von „Pulver mit Lonicera und Forsythia“ (Yinqiao san) und „Kleines Bupleurum-Dekokt“ (Xiao chaihu tang) zur gleichzeitigen Behandlung einer calor-venti-Affektion („Wind-Hitze“, fengre) der extima (Oberfläche, biao) und einer yang-minor-Symptomatik (Kleines Yang, shaoyang) angegeben. Ergänzend sind Arzneien zur Umwandlung und Kanalisierung von humor („Feuchtigkeit“, shi) und Isatidis radix (Banlangen) als im Rachenbereich antiviral wirkendes Arzneimittel hinzugefügt.

\subsubsection{Frühphase einer Affektion durch calor humidus („Feuchtigkeit-Hitze“, shire)}

Für dieses Stadium der Erkrankung wird eine Kombination von „Pulver mit Lonicera und Forsythia" (Yinqiao san) mit "Kleines Bupleurum-Dekokt“ (Xiao chaihu tang) und "Pulver mit Agastache zur Stärkung des geradläufigen Qi“ (Huoxiang zhengqi san) als Basisrezeptur angegeben. Trotz der angegebenen Musterdifferenzierung wird die Elimination von humor („Feuchtigkeit“, shi) hier vorwiegend durch aromatische Umwandlung von humor („Feuchtigkeit“, shi) und Kanalisierung bewerkstelligt. Ephedrae herba (Mahuang) dient der Entfaltung des qi pulmonale (Qi des Fk „Lunge“, feiqi).

\subsubsection{Calor humidus („Feuchtigkeit-Hitze“, shire) blockiert den o. pulmonalis} (Fk "Lunge“, fei) (shire yongfei 湿热隹肺)

Hier wird eine recht umfangreiche Rezeptur angegeben mit Arzneimitteln zur Elimination von calor humidus („Feuchtigkeit-Hitze“, shire), kombiniert mit solchen zur Regulierung des qi pulmonale (Qi des Fk „Lunge“, feiqi), zum Befeuchten, zum Erweichen von pituita calidae („Hitze-Schleim, retan), zur Verdauungserleichterung, zur Stützung von Xue und Yin sowie desinfizierende Zutaten.

\subsubsection{Blockade des o. pulmonalis (Fk "Lunge") durch Epidemisch-Toxisches" (yidu bifei 疫毒闭肺)}

In diesem gleichfalls umfangreichen Rezept kommen desinfizierende im Verein mit calor („Hitze“, re) klärenden Arzneien zur Anwendung, ergänzt durch Zutaten, die über den gekoppelten o. intestini crassi (Fk „Dickdarm“, dachang) calor („Hitze“, re) ableiten. Außerdem werden Arzneimittel zur Ausleitung von calor humidus („Feuchtigkeit-Hitze“, shire) und Blockaden lösende und Leitbahnen durchgängig machende Mittel hinzugefügt. Letztlich sorgen Ephedrae herba (Mahuang), Lepidii/Descurainiae semen (Tinglizi) und Platycodi radix (Jiegeng) für die Lösung des qi pulmonale (Qi des Fk „Lunge“, feiqi).

\subsubsection{Blockade im Inneren und Entweichen nach außen (neibi waituo 内闭外脱)}

Analog zu dem im „Hubei-Protokoll“ gleichnamigen Stadium bei einem drohenden terminalen Herz-Kreislauf-Versagen und komatösen Zuständen wird das folgende hinsichtlich Indikation und Verfügbarkeit der Bestandteile in deutschen Kliniken nicht realisierbare Konzept vorgeschlagen: „Das Dekokt gegen die vier Kontravektionen mit Ginseng radix“ (Sini jia renshen tang), „Pille des befriedeten Palastes mit Bovis calculus“ (Angong niuhuang wan), „Dekokt mit Ginseng und Aconitum“ (Shenfu tang). 


\subsubsection{Defizit von Qi und Yin sowie der oo. pulmonalis et lienalis (Fk "Lunge" und "Milz", fei pi)}

Zur Nachbehandlung nach überstandener schwerer Covid-19-Erkrankung werden Bestandteile von „Glehnia- und Ophiopogon-Dekokt“ (Shashen maidong tang) zur Stützung des Yin mit Arzneien zur Ergänzung des Qi, zur Regulation des qi pulmonale (Qi des Fk „Lunge“, feiqi) und zum Lösen von pituita („Schleim“, tan) kombiniert.

\subsubsection{Zusammenfassung und Kommentar}

Wie in dem Protokoll des Hubei Provincial Hospital liegt der Fokus auch hier auf der Behandlung von calor-Befunden („Hitze“, re). Die angegebenen Rezepturen tragen jeweils dem Umstand Rechnung, dass nahezu in jedem Stadium ein komplexes Krankheitsgeschehen zu behandeln ist.

\subsection{Anmerkungen zu den gesichteten Quellen}

Die Quellen, die in dieser Übersicht vorgestellt wurden, sind durchaus heterogen. Es liegen sowohl Therapieempfehlungen staatlicher Organe vor als auch Behandlungsregime verschiedener Kliniken, nicht zuletzt auch Erfahrungs- und Behandlungsberichte kleinerer Gruppen von Therapeuten der Chinesischen Medizin. Da die Behörden angesichts des sich dramatisch zuspitzenden Notstands in Wuhan und Umgebung Hilfskräfte jeder Art aus dem ganzen Land zusammengezogen hatten, arbeiteten offenbar auch mehrere Arbeitsgruppen der Chinesischen Medizin parallel und mehr oder weniger unabhängig voneinander. So sind möglicherweise die teilweise voneinander abweichenden Beobachtungen, diagnostischen Schlüsse und Therapiekonzepte zu erklären. Gleichwohl ist ein gewisser Konsens hinsichtlich der Deutung des Ablaufs der Covid-19-Erkrankung offensichtlich, der trotz der erkennbaren Unterschiede der beschriebenen Therapieansätze die Wichtigkeit hervorhebt, eine frühe Akkumulation von humor pituitae („Schleim-Feuchtigkeit“, tanshi), die besonders den o. pulmonalis (Fk „Lunge“, fei), aber auch die „Mitte“ blockiert, zu lösen und zu kanalisieren, gefolgt von der Klärung von calor-Toxischem („Hitze“, re) und Lösung von Blockaden im Stadium der schweren Pneumonie.

Es gab offenbar eine staatliche Anweisung, dass in allen medizinischen Einrichtungen in Wuhan die SARS-CoV-2-Infizierten ab dem 02.02.2020 mit chinesischen Arzneimitteln zu behandeln seien, so dass womöglich allein in Wuhan in wenigen Wochen ca. 60.000 Kranke mit Chinesischer Medizin behandelt worden sind. Berichten zufolge wurde in Einrichtungen, die primär keine Kliniken für Chinesische Medizin sind, ad hoc Abteilungen mit TCM-Therapeuten eingerichtet.

Einige der Therapieempfehlungen basieren auf Erfahrungen mit dem Ausbruch von SARS-CoV-1 im Jahr 2003. Wie wir inzwischen genauer wissen, gibt es jedoch mindestens zwei wesentliche Unterschiede der Eigenschaften des „alten“ und des aktuellen Corona-Virus:

$\rightarrow$ SARS-CoV-1 hat deutlich schneller zu pneumonischen Zuständen - und dann mit höherer Sterblichkeit in fast allen Altersgruppen - geführt als SARS-CoV-2. $\rightarrow$ Das aktuelle SARS-CoV-2 ist deutlich infektiöser und führt häufiger zu asymptomatischen oder milden Verläufen ohne Lungenbeteiligung.

Nach einem WHO-Bericht von 2004 über die Behandlung von Patienten, die an SARS-CoV-1 erkrankt und mit einer Kombination von westlicher und Chinesischer Medizin behandelt worden waren, wurden damals sowohl im frühen als auch im
Möglicher Grund für unterschiedliche Diagnosen und Therapien

Zur Behandlung von calor („Hitze“, re) 
Faktoren, die zu einem schweren Verlaufführen können späteren Verlauf gravierende calor-Befunde („Hitze“, re) festgestellt und behandelt. Einige der aktuell vorliegenden Behandlungsempfehlungen scheinen sich daran $\mathrm{zu}$ orientieren, doch gibt es ebenso Fachleute, die davor warnen, zu früh allzu kühlende Therapiestrategien anzuwenden.

\section{Eigene Überlegungen zum Verständnis der Covid-19-Erkran- kung und Vorschläge für an Krankheitsstadien orientierte Therapiekonzepte mit chinesischen Arzneimitteln}

3.1 Klinische Symptomatik bei Covid-19-Erkrankungen

$\rightarrow$ Initial oft keine oder mäßig ausgeprägte Symptomatik

$\rightarrow$ Viele blande verlaufende Infektionen

$\rightarrow$ Auffällige Häufung schwerer Verläufe bei Älteren und Geschwächten

Häufig: $\rightarrow$ Husten ohne oder mit wenig Auswurf

$\rightarrow$ Fieber ohne Schüttelfrost, evtl. leichte Kälteabneigung

$\rightarrow$ Schwäche, Ermüdbarkeit

$\rightarrow$ Kurzatmigkeit mit beschleunigter Atemfrequenz (duanqi)

$\rightarrow$ Atemnot, in schweren Fällen Ertrinkungsgefühl

$\rightarrow$ Gedunsene Zungenkörper, feuchte Zungenbeläge

$\rightarrow$ Pulse lubrici („schlüpfrig“, hua)

$\rightarrow$ Geruchs- und Geschmacksverlust

Teilweise: $\rightarrow$ Halsschmerzen

$\rightarrow$ Diarrhoe

$\rightarrow$ Schwindel

Selten: $\rightarrow$ Niesen, Schnupfen

$\rightarrow$ Schüttelfrost, plötzliches hohes Fieber als Initialsymptomatik

$\rightarrow$ Bei beatmungspflichtigen Patienten oft protrahierte respiratorische Insuffizienz

$\rightarrow$ Nach Abklingen der Akutsymptomatik persistierende restriktive Ventilationsstörungen

\section{2 Ätiologische Überlegungen}

3.2.1 Mögliche allgemeine prädisponierende Faktoren für das Eindringen der Erkrankung in die Lunge und schwere Verläufe:

$\rightarrow$ Alter

$\rightarrow$ Immunsystem schwächende Vorerkrankungen bzw. Therapien

$\rightarrow$ Vorerkrankungen des Atemtrakts und Herz-Kreislauf-Systems

$\rightarrow$ Stoffwechselerkrankungen

\subsubsection{Chinesische Medizin}

$\rightarrow$ Mangelhaft harmonisierte extima (Oberfläche, biao) (qi constructivum et defensivum, Bau- und Wehrenergie, yingqi weiqi) begünstigen Eindringen der Heteropathien (Schrägläufigkeiten, xie) in das Innere

$\rightarrow$ Defizientes Qi des o. renalis (FK „Niere“, shen) führt zur mangelhaften Unterstützung sowohl der Transformation der „Mitte“ als auch des qi defensivum (Wehrenergie, weiqi) des o. pulmonalis (Fk „Lunge“, fei)

$\rightarrow$ Vorbestehender innerer humor („Feuchtigkeit“, shi) bzw. vorbestehende innere pituita („Schleim“, tan) sowie Blockaden der „Mitte“ führen zur Akkumulation von humor pituitae („Schleim-Feuchtigkeit“, tanshi) im o. pulmonalis (Fk „Lunge“, fei) 


\subsection{Pathophysiologische Überlegungen}

\subsubsection{Natur des Pathogens Covid-19}

$\rightarrow$ Die Beobachtung, dass Infektionen oft zunächst keine oder nur milde Symptome verursachen, sprechen dafür, dass die SARS-CoV-2-Affektion offenbar kaum typische initiale Reaktionen hervorruft, die wir in der Chinesischen Medizin üblicherweise als „Stadium des qi defensivum“ („Stadium der Wehrenergie“, weiqi)“ (morbi temperati, „Wärme“-Erkrankungen, wenbing) bzw. „Stadium des yang maior" („Großes-Yang-Stadium“, taiyang) („Abhandlung über schädigende Kälte (algor laedens)“, Shanghan lun) bezeichnen würden. Somit gehören calor („Hitze“, re) bzw. algor („Kälte“, han) in der extima (Oberfläche, biao) hier nicht zu den dominierenden pathogenen Faktoren.

$\rightarrow \mathrm{Zu}$ den häufigeren früh geäußerten Symptomen gehören Schwäche und Müdigkeit, was für eine frühe Beteiligung einer humor-Heteropathie („Feuchtigkeit“-Schrägläufigkeit, shixie) mit Blockade der „Mitte“ spricht. Das häufige Auftreten von Diarrhoe spricht ebenfalls für eine Affektion des Qi der „Mitte“. Auffällig ist eine Beobachtung bei Covid-19-Erkrankten in Deutschland (Pressemitteilung Prof. H. Streeck, Virologie Universität Bonn), der zufolge ein hoher Anteil befragter Infizierter einen mehrtägigen Geschmacks- und Geruchsverlust beschrieb. Im Kontext der übrigen Symptomatik würde diese Beobachtung die Interpretation der Erkrankung als eine Akkumulation von Trübem unterstützen, die in diesen Fällen nicht nur die Transformation des Qi der „Mitte“ blockiert, sondern auch die Sinnesöffnungen.

$\rightarrow$ Im Einklang mit der in diversen Quellen geäußerten Auffassung ist bei der Covid-19-Erkrankung schon im Frühstadium primär von einer Affektion mit epidemischem humor-Toxischen („Feuchtigkeit“, shi) auszugehen [9].

\subsubsection{Dysharmoniemuster/Heteropathien (Schrägläufigkeiten, xie) im weiteren Verlauf}

$\rightarrow$ Die geschilderte typische Symptomatik im weiteren Verlauf sind Husten ohne Auswurf bzw. mit zähem, festsitzendem Schleim, Fieber, Schwäche und zunehmende Atemnot. Außerdem werden auch Völlegefühl, Appetitlosigkeit und Diarrhoe beschrieben. Bei einer Reihe von Erkrankten sind zudem nach zwischenzeitlicher Besserung Rückfälle mit erneuter respiratorischer Insuffizienz beobachtet worden. Die Atemnot wird offenbar oft als ein Ertrinkungsgefühl wahrgenommen.

$\rightarrow$ Die Angaben zur Symptomatik unterstützen die Annahme, dass das zentrale Dysharmoniemuster fortgeschrittener schwerer Verläufe der Covid-19Erkrankung die Akkumulation von humor („Feuchtigkeit“, shi) bzw. humor pituitae („Schleim-Feuchtigkeit", tanshi) ist, die den Fluss des qi pulmonale (Qi des Fk „Lunge“, feiqi) blockiert. Zudem spielt die mangelnde Transformation der Flüssigkeiten - sei es durch Defizit von Yang bzw. Qi und/oder durch humor-Überlastung („Feuchtigkeit“, shi) der „Mitte“ - nicht nur im Vorfeld, sondern auch in der Phase der akuten Erkrankung eine wichtige Rolle. Der von der Arbeitsgruppe um Liu Lihong vermutete Aspekt von eingetrockneter pituita („Schleim“, tan), die die Autoren für die beobachteten Rückfälle verantwortlich machen und wofür sie die Verwendung von pituita („Schleim“, tan) erweichenden Arzneien empfehlen, ist aus der Sicht des Autors dieser Arbeit als sekundärer Prozess aufgrund bereits einsetzender Entwicklung von calor („Hitze“, re) einzustufen.
Affektion der „Mitte“ 
Der primäre Krankheitsprozess

Mögliche Xue-Stasen

$\rightarrow$ Die divergierenden Einschätzungen verschiedener Quellen, ob die Akkumulation von humor („Feuchtigkeit“, shi) bzw. pituita („Schleim“, tan) calor-Aspekte („Hitze“, re) aufweist, lassen hauptsächlich zwei Schlussfolgerungen zu: Zum einen können konstitutionsbedingt die Reaktionen des Organismus zunächst unterschiedlich ausfallen. Überwiegend Yang- und Qi-schwache Personen dürften zumindest in der frühen pneumonischen Phase zunächst Akkumulationen von humor („Feuchtigkeit“, shi) bzw. pituita („Schleim“, tan) ohne calorBefunde („Hitze“, re) aufweisen, während stärkere Individuen früher calorZeichen („Hitze“, re) entwickeln. Falls sich die Erkrankung weiter verschlimmert, entwickeln beide Gruppen calor („Hitze“, re) bzw. calor-Toxisches („Hitze“,re) im Inneren. Zum anderen ist - konform mit den meisten Behandlungsempfehlungen aus China - die Akkumulation von humor („Feuchtigkeit“, shi) und Toxischem als primärer Krankheitsprozess anzusehen und die Entstehung von calor („Hitze“, re) als sekundäre Entwicklung. Sollten wir also präklinisch oder bereits stationär Covid-19-Erkrankte sehen, wird es essenziell sein, auf tatsächlich vorhandene calor-Zeichen („Hitze“, re) bei der Symptomatik und besonders dem Zungenbefund zu achten.

$\rightarrow$ Dem Zungenbefund und besonders dem Zungenbelag kommt bei der Verlaufsbeurteilung eine zentrale Bedeutung zu. Während die häufig geschwollenen Zungen im Verlauf oft zunächst blass erscheinen und die zunehmende Rötung die Entwicklung des calor-Aspekts („Hitze“, re) der Erkrankung widerspiegelt, weisen die meist im Frühstadium schon dicken Beläge auf die dann bereits bestehende Affektion des Inneren hin. Die in aller Regel feuchte Konsistenz des Belags ist ein klarer Hinweis auf die Existenz von humor („Feuchtigkeit“, shi) und die Farbe des Belags auf das Temperaturverhalten des Geschehens. Auch wenn der Belag sich im hinteren Bereich gelb zu verfärben und zu verdunkeln beginnt, ergibt sich aus dem Verbleiben von weißen bzw. gräulichen Belaganteilen in mittleren und vorderen Abschnitten der Zunge die Notwendigkeit der Umwandlung von humor („Feuchtigkeit“, shi) mit scharfen und aromatischen Arzneien. Sobald der Belag vollkommen eingetrocknet und gelb oder braun verfärbt ist, ist das Stadium erreicht, in dem Stagnation von Toxischem und calor („Hitze“, re) das Krankheitsgeschehen dominieren.

$\rightarrow$ In den Protokollen aus dem Hubei- und Wuhan-Hospital sind für die Phase der Stagnation von Toxischem auch Arzneimittel zur Behandlung von Xue-Stasen angegeben. Eingedenk der klinischen Beobachtungen, dass die Patienten teilweise bereits in frühen Stadien der Pneumonie analog zur stark reduzierten Sauerstoffsättigung Lippen- und Akrozyanose sowie livide verfärbte Zungenkörper aufweisen, verdient möglicherweise eine besondere Berücksichtigung von Xue-Stasen in der Therapie mehr Beachtung als bisher üblicherweise diskutiert worden ist. Hinweise auf Herzmuskelbeteiligungen wie auffällige Troponin-Tests sind weitere Fingerzeige darauf, dass nicht nur der Fluss des Qi, sondern auch der des Xue im Brustbereich affiziert ist.

\subsubsection{Abklingphase und Nachbehandlung}

Die nach dem Überstehen der entzündlichen pneumonischen Phase oft persistierende respiratorische Insuffizienz mit fortbestehender Beatmungspflichtigkeit und die restriktiven Ventilationsstörungen nach Abklingen der Akutphase können als Defizite von Qi und Yin des o. pulmonalis (Fk „Lunge“, fei) sowie als Persistenz von eingetrockneter pituita („Schleim“, tan) interpretiert werden. 


\subsection{Therapieprinzipien}

\subsubsection{Prävention}

$\rightarrow$ Stützen der Transformationsfunktion der „Mitte“ und des o. renalis (Fk „Niere“, shen) durch diätetische und medikamentöse Maßnahmen

$\rightarrow$ Qi stützen, extima (Oberfläche, biao) stabilisieren, Pathogene Faktoren abwehren

3.4.2 Initiales Stadium - Affektion der extima (Oberfläche, biao)

$\rightarrow$ Lösen der extima (Oberfläche, biao) und Eliminieren der beobachteten Heteropathien (Schrägläufigkeiten, xie) unter besonderer Berücksichtigung von:

$\rightarrow$ Unterstützen und Harmonisieren der „Mitte“

$\rightarrow$ Umwandeln und Kanalisieren von humor („Feuchtigkeit“, shi)

\subsubsection{Schwere Verläufe - Affektion der intima (Inneres, k), pneumonische} Verläufe

$\rightarrow$ Umwandeln und Kanalisieren von humor pituitae („Schleim-Feuchtigkeit“, tanshi) im oberen und mittleren Calorium (Wärmebereich, jiao)

$\rightarrow$ Entfalten des qi pulmonale (Qi des Fk „Lunge“, feiqi)

$\rightarrow$ Unterstützen und Harmonisieren der "Mitte“

$\rightarrow$ Evtl. Unterstützen/Wärmen des Yang des o. renalis (Fk „Niere“, shen)

$\rightarrow$ Evtl. Erweichen und Auflösen von „trockener“ pituita („trockener Schleim“, zaotan)

$\rightarrow$ Lösen der Stagnation von Toxischem

$\rightarrow$ Klären vorhandener calor-Aspekte („Hitze“, re)

\subsubsection{Abkling- und Nachbehandlungsphase}

$\rightarrow$ Stützen von defizitärem Qi bzw. Yin des o. pulmonalis (Fk „Lunge“, fei)

$\rightarrow$ Lösen und Erweichen verbliebener pituita-Befunde („Schleim“, tan)

$\rightarrow$ Eliminieren verbliebener pathogener Restfaktoren

\subsection{Mögliche Therapiekonzepte}

\subsubsection{Präventionsphase}

a) Bei Tendenz zu humor pituitae („Schleim-Feuchtigkeit“, tanshi) und Blockaden der "Mitte“

$\rightarrow$ Stützen und Harmonisieren der „Mitte“, humor („Feuchtigkeit“, shi) eliminierend: „Dekokt der sechs Edlen“ (Liu junzi tang), „Pulver mit Ginseng, Poria und Atractylodis macrocephalae“ (Shenling baizhu san), „Pulver mit Agastache zur Stärkung des geradläufigen Qi“ (Huoxiang zhengqi san)

b) Bei mangelhafter Erwärmung durch defizientes Yang

$\rightarrow$ Stützen und Erwärmen der „Mitte“: „Die Mitte regulierende Pille“ (Lizhong wan), „Die Mitte regulierende Aconit-Pille“(Fuzi lizhong wan)

$\rightarrow$ Stützen des Qi, Stabilisieren der extima (Oberfläche, biao): „Pulver gegen Wind aus Jade“ (Yu pingfeng san), eventuell + „Pulver mit Lonicera und Forsythia“ (Yinqiao san) oder Einzelarzneien: + Lonicerae flos (Jinyinhua), Isatidis radix (Banlangen), Dryopteridis rhizoma (Guanzhong)

$\rightarrow$ Die Rezepturen können jeweils als Module betrachtet werden, mit denen individuelle konstitutionelle Tendenzen behandelt werden können.

$\rightarrow$ Kombinationen mit dem „Pulver gegen Wind aus Jade“ (Yu pingfeng san) werden sicherlich im Gegensatz zum SARS-CoV-1-Ausbruch kaum Infektionen verhindern, können jedoch dazu beitragen, die Erkrankung
Das Vorgehen bei schweren Verläufen

Mögliche Wirkung des „Pulvers gegen

Wind aus Jade"(Yu pingfeng san) 
durch die Infektion entweder ganz abzuwehren oder das Eindringen der Erkrankung in die Lunge zu vermeiden oder wenigstens zu verzögern.

$\rightarrow$ Cave Astragali radix (Huangqi): Vorsicht bei Personen mit Autoimmunerkrankungen und nach John K. Chen bei Immunsupprimierten.

\subsubsection{Initiale Stadien - Affektion der extima (Oberfläche, biao) -}

bzw. leichte Erkrankungen

$\rightarrow$ Anzeichen für Blockade der "Mitte“ mit Bildung von humor („Feuchtigkeit“, shi): „Pulver mit Agastache zur Stärkung des geradläufigen Qi“ (Huoxiang zhengqi san)

Da die Covid-19-Erkrankung in erster Linie als eine Affektion epidemischen humor-Toxischem („Feuchtigkeit“, shi) angesehen wird, ist das „Pulver mit Agastache zur Stärkung des geradläufigen Qi“ (Huoxiang zhengqi san) aus der Sicht des Autors die wichtigste Grundrezeptur zur Behandlung der frühen und mittleren Stadien der Erkrankung.

$\rightarrow$ Bei calor-venti-Zeichen („Wind-Hitze“, fengre), Husten, Halsschmerzen: „Pulver mit Lonicera und Forsythia“ (Yinqiao san)

$\rightarrow$ Bei Anzeichen von yang-minor-Symptomatik (Kleines Yang, shaoyang): „Kleines Bupleurum-Dekokt“ (Xiao chaihu tang)

$\rightarrow$ Jeweils mit eventuell geeigneten Modifikationen

$\rightarrow$ Bei Zeichen von algor venti („Wind-Kälte“, fenghan), Fieber, Schüttelfrost ohne Schweißbildung (selten bei Covid-19): „Ephedra-Dekokt“ (Mahuang tang)

$\rightarrow$ Bei Zeichen von algor venti („Wind-Kälte“, fenghan), mäßiger Kälteabneigung mit Schwitzen: „Dekokt mit Cinnamomum“ (Guizhi tang)

\subsection{Stadien mit Lungenbeteiligung bzw. Pneumonie - Einzelarzneien}

Das erklärte Therapieziel bei Erkrankten mit beginnender Lungenbeteiligung sollte sein, die Ausprägung interstitieller Infiltrate mit massiven alveolären Exsudationen zu vermeiden bzw. wenigstens abzumildern, um der Entwicklung einer terminalen beatmungspflichtigen respiratorischen Insuffizienz entgegenzuwirken. Das Anfangsstadium der sich entwickelnden Pneumonie dürfte in der Regel (ob noch ambulant oder in der frühstationären Behandlungsphase) die letzte Gelegenheit sein, den Erkrankungsverlauf mit Hilfe der Chinesischen Medizin noch positiv beeinflussen zu können. Aus der Sicht der Chinesischen Medizin spielt in dieser Phase nach allgemeiner Übereinstimmung folgendes Krankheitsmuster die wichtigste Rolle und sollte im Zentrum der therapeutischen Anstrengungen stehen:

\subsubsection{Akkumulation von humor („Feuchtigkeit", shi) bzw. pituita} ("Schleim", tan) im o. pulmonalis (Fk "Lunge", fei) und der "Mitte" mit Blockade des qi pulmonale (Qi des Fk "Lunge", feiqi) tan) umwandelnde Arzneien sowie Diuretika. Als besonders wirkungsvoll können dabei folgende Arzneimittel gelten:

$\rightarrow$ Magnoliae cortex (Houpo): humor pituitae („Schleim-Feuchtigkeit“, tanshi) umwandelnd, Qi regulierend

$\rightarrow$ Atractylodis rhizoma (Cangzhu): humor („Feuchtigkeit“, shi) umwandelnd, o. lienalis (Fk „Milz“, pi) stärkend

$\rightarrow$ Agastachis herba (Huoxiang): Akkumulation durch humor („Feuchtigkeit“, shi) auflösend, „Mitte“ harmonisierend 
$\rightarrow$ Eupatorii herba (Peilan): humor pituitae („Schleim-Feuchtigkeit“, tanshi) umwandelnd

$\rightarrow$ Sinapis albae semen (Baijiezi):pituita („Schleim“, tan) umwandelnd, Qi regulierend

$\rightarrow$ Lepidii/Descurainiae semen (Tinglizi): Akkumulation von humorpituitae(,SchleimFeuchtigkeit“, tanshi) im o. pulmonalis (Fk „Lunge“, fei) lösend, Qi regulierend

$\rightarrow$ Pinelliae rhizoma (Banxia): humor („Feuchtigkeit“, shi) und pituita („Schleim“, tan) umwandelnd, Qi regulierend

$\rightarrow$ Platycodi radix (Jiegeng): pituita („Schleim“, tan) umwandelnd, qi pulmonale (Qi des Fk „Lunge“, feiqi) lösend, Meldearznei o. pulmonalis (Fk „Lunge“, fei)

$\rightarrow$ Perillae fructus (Zisuzi): pituita („Schleim“, tan) umwandelnd, qi pulmonale (Qi des Fk „Lunge“, feiqi) lösend

$\rightarrow$ Poria (Fuling): diuretisch, kanalisierend, „Mitte“ stützend

\subsubsection{Ferner Arzneien zum Erweichen von "trockener" pituita (,trockener} Schleim", zaotan) und Arzneimittel, die auch Aspekte von calor humidus ("Feuchtigkeit-Hitze“, shire) behandeln

$\rightarrow$ Trichosanthis semen (Gualouren): pituita („Schleim“, tan) erweichend, „BrustQi“lösend

$\rightarrow$ Fritillariae thunbergii bulbus (Zhebeimu): heiße und zähe pituita („Schleim“, tan) lösend

$\rightarrow$ Scutellariae radix (Huangqin): calor („Hitze“, re) bzw. calor humidus („Feuchtigkeit-Hitze", shire) im oberen Calorium (oberer Wärmebereich, shangjiao) klärend

$\rightarrow$ Coicis semen (Yiyiren): humor („Feuchtigkeit“, shi) bzw. calor humidus („Feuchtigkeit-Hitze“, shire) klärend, diuretisch, „Mitte“ stützend

$\rightarrow$ Benincasae semen (Dongguazi): calor humidus („Feuchtigkeit-Hitze“, shire) und pituita („Schleim“, tan) klärend

$\rightarrow$ Coptidis rhizoma (Huanglian): in einigen Quellen im Kontext von „Kleines Dekokt gegen Vernichtungsgefühl in der Brust“ (Xiao xianxiong tang) angegeben, damit Blockaden im Thorax lösend

\subsubsection{Außerdem wichtig: Qi regulierende Arzneimittel, die beim Umwan- deln und Kanalisieren von humor („Feuchtigkeit", shi) bzw. pituita ("Schleim", tan) assistieren}

$\rightarrow$ Citri reticulatae pericarpium (Chenpi): humor pituitae („Schleim-Feuchtigkeit“, tanshi) umwandelnd, Stauungen beidseits des Zwerchfells lösend

$\rightarrow$ Arecae pericarpium (Dafupi): abschwellend, diuretisch, Qi regulierend

$\rightarrow$ Citri grandis exocarpium rubrum (Huajuhong): humor pituitae („SchleimFeuchtigkeit“, tanshi) umwandelnd, „Brust-Qi“ lösend

$\rightarrow$ Allii macrostemonis bulbus (Xiebai): Blockaden des Yang-Qi und Akkumulation von Trübem lösend

$\rightarrow$ Pheretima (Dilong): calor („Hitze“, re) klärend, (Netz-)Leitbahnen freimachend

\subsubsection{Arzneimittel zum Lösen von Blockaden des Energieflusses in der}

\section{Brust und der Atmung}

$\rightarrow$ Ephedrae herba (Mahuang): Während des aktuellen Covid-19-Ausbruchs in Wuhan wurde Ephedrae herba (Mahuang) offenbar als eine der wichtigsten Arzneien in vielen Rezepturen verwendet - dabei weniger unter dem Aspekt ihres extima (Oberfläche, biao) freimachenden Effekts, sondern hauptsächlich wegen ihrer lösenden Wirkung auf das qi pulmonale (Qi des Fk „Lunge“, feiqi)

Humor („Feuchtigkeit“, shi) umwandelnde Arzneimittel 
Eine wichtige Arzneimittelkombination

Bestätigte Wirkung bei Pneumonien und die Atmung sowie die abschwellende Wirkung. Für diesen Effekt ist Ephedrae herba (Mahuang) in Honig geröstet (Mizhi mahuang) geeigneter, sofern verfügbar. In diesem Kontext wird Ephedrae herba (Mahuang) meist zusammen mit Gypsum fibrosum (Shigao) eingesetzt.

$\rightarrow$ Armeniacae semen (Xingren): qi pulmonale (Qi des Fk „Lunge“, feiqi) und Husten lösend. Das Mittel wirkt mit Ephedrae herba (Mahuang) in dieser Hinsicht synergistisch. Beide werden als eine der meistverwendeten Arzneikombinationen in den hier gesichteten Quellen angegeben. Zumeist werden die Arzneien weniger im Kontext von „Ephedra-Dekokt“ (Mahuang tang) verwendet, also nicht primär zur Entfaltung der extima (Oberfläche, biao), sondern vorwiegend im Kontext von „Dekokt der drei Ungebundenen“ (Sariao tang), „Baldachin-Pulver“ (Huagai san), „Dekokt mit Ephedra, Prunus armeniaca, Gypsum und Glycyrrhiza“ (Maxing shigan tang) bzw. „Dekokt mit Ephedra, Prunus armeniaca, Coix und Glycyrrhiza" (Maxing yigan tang), somit primär zur Behandlung des Inneren des o. pulmonalis (Fk „Lunge“, fei).

$\rightarrow$ Die Kombination von Ephedrae herba (Mahuang) und Armeniacae semen (Xingren) ist in Wuhan auch in Rezepturen zur Behandlung von calor-Mustern („Hitze“, re) wie calor humidus („Feuchtigkeit-Hitze“, shire) und calor-Toxisches („Hitze“, re) zur Anwendung gekommen und gehört zu den wichtigsten zur Behandlung der "Verzweigung" (cacumen, biao) der Erkrankung, der Blockade des qi pulmonale (Qi des Fk „Lunge“, feiqi).

$\rightarrow$ Platycodi radix (Jiegeng): pituita („Schleim“, tan) umwandelnd, qi pulmonale (Qi des Fk „Lunge“, feiqi) lösend, Meldearznei o. pulmonalis (Fk „Lunge“, fei)

$\rightarrow$ Mori cortex (Sangbaipi): calor („Hitze“, re) und Flüssigkeiten drainierend, Husten stillend

\subsubsection{Arzneien zum Klären von calor-Toxischem („Hitze“, re)}

Unter Berücksichtigung der vorherrschenden Expertenmeinung, dass sich primär "toxischer humor (,Feuchtigkeit', shi) entwickelt und die Entstehung von calor (,Hitze', re) eine sekundäre Folge der Stagnation von Toxischem ist“, sind folgende Arzneien zur Behandlung von calor-Toxischem („Hitze“, re) geeignet:

$\rightarrow$ Lonicerae flos (Jinyinhua): Zählt zu den am häufigsten empfohlenen Arzneien bei SARS-CoV-2-Infektionen, von der Phase der Infektionsprävention bis hin zu schweren pneumonischen Stadien. Lonicerae flos (Jinyinhua) hat sich in chinesischen Studien zur Infektionsprophylaxe als sehr effektiv erwiesen und klärt calor-Toxisches („Hitze“, re) nicht nur an der extima (Oberfläche, biao), sondern auch in tiefen Schichten (nach morbi temperati, "Wärme“-Erkrankungen, wenbing). Zudem wird dem Mittel eine antivirale Wirkung zugesprochen.

$\rightarrow$ Forsythiae fructus (Lianqiao): synergistisch mit Lonicerae flos (Jinyinhua) wirkend

Beide Arzneien können in unterschiedlichen Stadien und Schweregraden der Erkrankung eingesetzt werden.

$\rightarrow$ Houttuyniae herba cum radice (Yuxingcao): calor-Toxisches („Hitze“, re) im o. pulmonalis (Fk „Lunge“, fei) klärend. Die Arznei ist in China, u.a. mit Platycodi radix (Jiegeng), mit Erfolg auf ihre Effektivität bei Pneumonien untersucht worden und in der aktuellen Corona-Krise in mehreren Therapieprotokollen zur Behandlung der pneumonischen Phase der Covid-19-Erkrankung enthalten.

$\rightarrow$ Belamcandae rhizoma (Shegan): klärt calor-Toxisches („Hitze“, re), löst pituita („Schleim“, tan) und qi pulmonale (Qi des Fk „Lunge“, feiqi) 
$\rightarrow$ Dryopteridis rhizoma (Guanzhong): Antiparasitikum mit calor-Toxisches („Hitze“, re) klärenden und antiviralen Eigenschaften

\subsubsection{Kühlende bzw. calor („Hitze“, re) klärende Arzneimittel}

$\rightarrow$ Gypsum fibrosum (Shigao): calor („Hitze“, re) im o. pulmonalis (Fk „Lunge“,fei) klärend, Toxisches klärende Wirkung anderer Arzneien unterstützend. Das Mittel wird in verschiedenen Stadien von Covid-19 zusammen mit Ephedrae herba (Mahuang) und Armeniacae semen (Xingren) im Kontext von „Dekokt mit Ephedra, Prunus armeniaca, Gypsum und Glycyrrhiza" (Maxing shigan tang) eingesetzt, oder im Kontext von „Dekokt des weißen Tigers“ (Baihu tang) mit folgenden Arzneien:

$\rightarrow$ Anemarrhenae rhizoma (Zhimu): calor („Hitze“, re) klärend, Yin erhaltend

$\rightarrow$ Phragmitis rhizoma (Lugen): calor („Hitze“, re) klärend, befeuchtend, ohne humor („Feuchtigkeit“, shi) zu begünstigen

$\rightarrow$ Trichosanthis radix (Tianhuafen): calor („Hitze“, re) und Toxisches klärend, befeuchtend

$\rightarrow$ Rhei rhizoma (Dahuang): Ableitung von calor („Hitze“, re) und Toxischem über den gekoppelten orbis (Funktionskreis, zangfu)

Obwohl in den meisten Stadien der Erkrankung die Akkumulation von humor pituitae („Schleim-Feuchtigkeit“, tanshi) behandelt werden muss, sind bei zunehmender calor-Entwicklung („Hitze“, re) die Säfte und das Yin von Austrocknung bedroht und somit Arzneien, die dem Erhalt des Yin und der Befeuchtung dienen, angezeigt.

\subsubsection{Arzneien zum Erwärmen}

Die Indikation von erwärmenden Arzneimitteln bei an Covid-19-Erkrankten wird in den vorliegenden Quellen unterschiedlich beurteilt und sollte von den klinischen Zeichen abhängig gemacht werden.

$\rightarrow$ Aconiti radix lateralis praeparata (Fuzi): Wird in den meisten Quellen ausschließlich für den Zustand eines hochgradig geschwächten Qi und kollabierenden Yang, also bei akut lebensbedrohlicher Herz-Kreislauf-Insuffizienz, zusammen mit Ginseng radix (Renshen) gegeben, und dann in China i.d.R. intravenös, was bei uns nicht realisierbar ist. Von einigen Autoren wird Aconiti radix lateralis praeparata ( Fuzi) - etwa im Kontext von „Die Mitte regulierende Aconit-Pille“ (Fuzi lizhong wan) - vor allem in der Prävention empfohlen, um bei einem geschwächten Yang des o. lienalis (Fk „Milz“, pi) die Voraussetzung für eine funktionierende Flüssigkeitstransformation zu gewährleisten.

$\rightarrow$ Zingiberis rhizoma (Ganjiang): „Mitte“ und o. pulmonalis (Fk „Lunge“, fei) wärmend, pituita („Schleim“, tan) umwandelnd

$\rightarrow$ Asari radix (Xixin): o. pulmonalis (Fk „Lunge“, fei) wärmend, pituita („Schleim“, tan) lösend

\subsubsection{Arzneien zum Lösen von Xue-Stasen}

Wie im Abschnitt zu den pathophysiologischen Überlegungen bereits angedeutet, kann bei Zeichen wie Zyanose und lividem Zungenkörper die Verschreibung von Xue-Stasen beseitigenden Arzneien angezeigt sein:

$\rightarrow$ Persicae semen (Taoren): Xue-Stasen u.a. im Thorax zerteilend

$\rightarrow$ Achyranthis bidentatae radix (Niuxi): Xue-Stasen lösend, calor („Hitze“, re) und Xue aus dem oberen Calorium (oberer Wärmebereich, shangjiao) herabführend
Befeuchtende und das Yin bewahrende Arzneien

Möglichkeiten für hiesige Therapeuten 
$\rightarrow$ Rhei rhizoma (Dahuang): Xue-Stasen zerstreuend, calor („Hitze“, re) und Akkumulation ableitend

$\rightarrow$ Curcumae longae tuber (Yujin): Xue-Stasen im Thorax zerteilend

$\rightarrow$ Polygoni cuspidati rhizoma (Huzhang): Xue-Stasen lösend, humor („Feuchtigkeit“, shi) und pituita („Schleim“, tan) umwandelnd, Toxisches klärend, calor („Hitze“, re) ableitend

\subsection{Praktische Umsetzung von Behandlungskonzepten für die „pneumonischen Stadien“ von Covid-19}

Je nach dem persönlichen Tätigkeitsbereich stellt sich für Therapeuten der Chinesischen Medizin die Frage, in welchem Stadium der Erkrankung Covid-19-Patienten überhaupt therapeutisch erreichbar sind. Nach der anfänglichen Praxis, Personen mit gesicherter Infektion frühzeitig stationär aufzunehmen, geht - je nach betroffener Region - der Trend teilweise gezwungenermaßen dahin, Patienten erst mit zunehmender Atemnotsymptomatik stationär zu behandeln, so dass zunehmend Erkrankte mit bereits manifester Pneumonie ambulant zu behandeln sein werden. Somit kann sich auch den Therapeuten, die keinen Zugriff auf klinische Behandlungsoptionen haben, die Chance bieten, in dieser Zeit einer gravierenden Verschlechterung des Allgemeinzustands entgegenzuwirken, indem durch frühzeitige Elimination der Akkumulation von humor („Feuchtigkeit“, shi) der Entstehung von manifester Stagnation von Toxischem mit calor („Hitze“, re) entgegengewirkt wird, um somit der kritischen Reduzierung der alveolären Funktion mit konsekutiver Notwendigkeit einer maschinellen Beatmung vorzubeugen. Angesichts der leider stark reduzierten Überlebenswahrscheinlichkeit beatmeter Covid-19-Patienten ist die beschriebene frühzeitige Therapie bei noch milder Symptomatik als essenziell anzusehen.

\subsection{Konzeptvorschläge für die „pneumonischen Stadien“ von Covid-19-Erkrankungen}

Grundsätzlich sollte sich die Therapie von Covid-19-Patienten getreu den Grundsätzen der Chinesischen Medizin an den erkennbaren Zeichen und somit dem jeweiligen Stadium der Erkrankung orientieren. „Universalspezifische Rezepte“ mindern die Erfolgschancen der Therapie und vergrößern das Potenzial unerwünschter Nebenwirkungen. Die vorgestellten Konzepte basieren auf dem aktuellen Literaturkonsens und sind als Vorschläge zur Therapie zu betrachten, die der jeweiligen klinischen Situation anzupassen sind. Die Konzepte zielen auf präklinische Stadien mit bereits begonnener Lungenbeteiligung bis hin zu klinisch behandlungsbedürftigen Stadien, in denen keine bzw. noch keine invasive Beatmung notwendig ist.

\subsection{Wichtige Grundkonstellationen pneumonischer Stadien der Erkrankung}

Die Symptome in frühen Stadien bei Lungenbeteiligung
3.9.1 Akkumulation von humor pituitae ("Schleim-Feuchtigkeit“, tanshi) behindert Entfaltung des qi pulmonale (Qi des Fk "Lunge", feiqi)

- Frühe Stadien mit Lungenbeteiligung ohne Anzeichen von calor („Hitze“, re)

- Symptome

$\rightarrow$ Husten ohne oder mit wenig zähem farblosem Schleim, leichte Atemnot, Druckgefühl 
$\rightarrow$ Mäßiges Fieber ohne Hitzeempfinden, evtl. Frösteln, Schwäche, Müdigkeit

$\rightarrow$ Evtl. Unwohlsein, Appetitlosigkeit, Durchfall, Geschmacks- und Geruchsverlust

$\rightarrow$ Zunge: Körper blass, gedunsen, Belag feucht und weiß bzw. grau und schmierig

$\rightarrow$ Pulse: lubrici („schlüpfrig“, hua), besonders an cun-Position rechts

- Therapieprinzipien

$\rightarrow$ Umwandeln und Kanalisieren von pituita („Schleim“, tan) und humor („Feuchtigkeit“, shi)

$\rightarrow$ Entfalten des qi pulmonale (Qi des Fk „Lunge“, feiqi), siehe Kasten rechts

$\rightarrow$ Stützen und Harmonisieren der „Mitte“

- Covid-19 A: Rezeptur zur Elimination von humor („Feuchtigkeit“, shi) und Entfaltung des qi pulmonale (Qi des Fk „Lunge“, feiqi), (s. Kasten rechts)

- Das Rezept ist eine modifizierte Kombination folgender Rezepturen: „Pulver mit Agastache zur Stärkung des geradläufigen Qi“ (Huoxiang zhengqi san), „Ephedra-Dekokt“ (Mahuang tang), „Dekokt mit Lepidium und Jujuba zur Zerstreuung des Fk Lunge (o. pulmonalis)“ (Tingli dazao xiefei tang)

- Modifikationen

$\rightarrow$ Ausgeprägte Schwäche mit Durchfall: + Atractylodis macrocephalae rhizoma (Baizhu)

$\rightarrow$ Festsitzender bzw. gelber Schleim: + Trichosanthis semen (Gualouren), Fritillariae thunbergii bulbus (Zhebeimu)

$\rightarrow$ Bei yang-minor-Symptomatik (Kleines Yang, shaoyang) wie bitterer Mundgeschmack, Wechsel von Frösteln und Wärme: + Bupleuri radix (Chaihu), Scutellariae radix (Huangqin), Pinelliae rhizoma (Banxia)

$\rightarrow$ Schmerzen im Rachen: + Lonicerae flos (Jinyinhua), Forsythiae fructus (Lianqiao), Isatidis radix (Banlangen)

$\rightarrow$ Stockende Verdauung, Appetitverlust: + Crataegi fructus (Shanzha), Hordei fructus germinatus (Maiya), Massa medicata fermentata (Shenqu)

$\rightarrow$ Calor-Zeichen („Hitze“, re): + Gypsum fibrosum (Shigao)

$\rightarrow$ Verstärkte Atemnot, thorakales Druckgefühl: + Trichosanthis semen (Gualouren)

$\rightarrow$ Zur Förderung der Kanalisierung: + Pheretima (Dilong)

\subsubsection{Calor humidus („,Feuchtigkeit-Hitze“, shire) affiziert den o. pulmonalis (Fk "Lunge", fei)}

- Frühe und mittlere Stadien der Pneumonie mit humor- („Feuchtigkeit“, shi) und calor-Zeichen („Hitze“, re)

- Symptome

$\rightarrow$ Husten ohne oder mit wenig zähem gelblichem Schleim, leichte Atemnot, Druckgefühl

$\rightarrow$ Mäßiges Fieber, Schwäche, Schweregefühl

$\rightarrow$ Evtl. Unwohlsein, Appetitlosigkeit, Defäkation erschwert bei weichem Stuhl

$\rightarrow$ Zunge: blass oder gerötet, Belag gelblich und schmierig

$\rightarrow$ Pulse: lubrici („schlüpfrig“, hua), evtl. celeri („,beschleunigt“, shu)

$\rightarrow$ Bei evtl. ähnlicher Symptomatik wie im vorherigen Fall kann die Farbe des Expektorats, des Zungenkörpers und des Belags bei der Beurteilung des Ausmaßes des calor-Aspekts („Hitze“, re) entscheidend sein.

- Therapieprinzip:

$\rightarrow$ Umwandeln und Kanalisieren von humor („Feuchtigkeit“, shi)

\section{Rezeptur zur Elimination von} humor („Feuchtigkeit“, shi) und Entfaltung des qi pulmonale (Qi des Fk „Lunge“, feiqi) Ephedrae herba (Mahuang) $6 \mathrm{~g}$ Armeniacae semen (Xingren) $9 \mathrm{~g}$ Agastachis herba (Huoxiang) $9 \mathrm{~g}$ Magnoliae cortex (Houpo) $9 \mathrm{~g}$ Atractylodis rhizoma (Cangzhu) $9 \mathrm{~g}$ Lepidii/Descurainiae semen (Tinglizi) $9 \mathrm{~g}$

Platycodi radix (Jiegeng) $9 \mathrm{~g}$

Pinelliae rhizoma (Banxia) $9 \mathrm{~g}$

Citri reticulatae pericarpium

(Chenpi) $6 \mathrm{~g}$

Poria (Fuling) $9 \mathrm{~g}$

Arecae pericarpium (Dafupi) $6 \mathrm{~g}$

Sinapis albae semen (Baijiezi) $9 \mathrm{~g}$

Zingiberis rhizoma recens (Shengjiang) $6 \mathrm{~g}$

Jujubae fructus (Dazao) $3 \mathrm{~g}$ 


\section{Rezeptur zum Umwandeln von humor („Feuchtigkeit“, shi), zum Ausleiten von calor humidus („Feuchtigkeit-Hitze“, shire) und zum Lösen des o. pulmonalis (Fk „Lunge“, fei) \\ Ephedrae herba (Mahuang) $6 \mathrm{~g}$ Armeniacae semen (Xingren) $9 \mathrm{~g}$ Coicis semen (Yiyiren) $9 \mathrm{~g}$ Magnoliae cortex (Houpo) $9 \mathrm{~g}$ Pinelliae rhizoma (Banxia) $9 \mathrm{~g}$ Benincasae semen (Dongguazi) $9 \mathrm{~g}$ Scutellariae radix (Huangqin) $9 \mathrm{~g}$ Poria (Fuling) $9 \mathrm{~g}$ \\ Lepidii/Descurainiae semen (Tinglizi) $9 \mathrm{~g}$ Amomi fructus rotundus (Baidoukou) $9 \mathrm{~g}$ Glycyrrhizae radix (Gancao) $6 \mathrm{~g}$}

\section{Rezeptur zum Eliminieren von} calor („Hitze“, re), humor („Feuchtigkeit“, shi) und Stagnation von Toxischem zur Entfaltung des $o$. pulmonalis (Fk „Lunge“, fei) Ephedrae herba (Mahuang) $6 \mathrm{~g}$ Armeniacae semen (Xingren) $9 \mathrm{~g}$ Gypsum fibrosum (Shigao) $30 \mathrm{~g}$ Glycyrrhizae radix (Gancao) $3 \mathrm{~g}$ Lepidii/Descurainiae semen (Tinglizi) $10 \mathrm{~g}$ Platycodi radix (Jiegeng) $10 \mathrm{~g}$ Trichosanthis semen (Gualouren) $9 \mathrm{~g}$ Agastachis herba (Huoxiang) $10 \mathrm{~g}$ Magnoliae cortex (Houpo) $10 \mathrm{~g}$ Poria (Fuling) $15 \mathrm{~g}$ Lonicerae flos (Jinyinhua) $15 \mathrm{~g}$ Forsythiae fructus (Lianqiao) $15 \mathrm{~g}$ Houttuyniae herba cum radice (Yuxingcao) $30 \mathrm{~g}$ Persicae semen (Taoren) $9 \mathrm{~g}$ Rhei rhizoma (Dahuang) $6 \mathrm{~g}$ $\rightarrow$ Ausleiten von calor humidus („Feuchtigkeit-Hitze“, shire) und Stagnation von Toxischem

$\rightarrow$ Entfalten des qi pulmonale (Qi des Fk „Lunge“, feiqi)

- Covid-19 B: Rezeptur zum Umwandeln von humor („Feuchtigkeit“, shi), zum Ausleiten von calor humidus („Feuchtigkeit-Hitze“, shire) und zum Lösen des $o$. pulmonalis (Fk „Lunge“, fei), (siehe Kasten links)

- Das Rezept ist eine modifizierte Kombination folgender Rezepturen: „Dekokt mit Ephedra, Prunus armeniaca, Coicis und Glycyrrhiza“" (Maxing yigan tang), „Dekokt der drei Samen“ (Sanren tang)

- Modifikationen

$\rightarrow$ Ausgeprägte Atemnot: + Platycodi radix (Jiegeng), Trichosanthis semen (Gualouren)

$\rightarrow$ Ausgeprägte calor-Zeichen („Hitze“, re), Obstipation: + Gypsum fibrosum (Shigao), Persicae semen (Taoren), Achyranthis bidentatae radix (Niuxi) bzw. + ,Dekokt des weißen Tigers“ (Baihu tang)

$\rightarrow$ Calor-Zeichen („Hitze“, re) mit Trockenheit: + Phragmitis rhizoma (Lugen), Trichosanthis radix (Tianhuafen)

$\rightarrow$ Hohes Fieber, reduzierter Allgemeinzustand: + Houttuyniae herba cum radice (Yuxingcao), Lonicerae flos (Jinyinhua), Forsythiae fructus (Lianqiao)

$\rightarrow$ Dominanz von humor-Zeichen („Feuchtigkeit“, shi): + Agastachis herba (Huoxiang), Atractylodis rhizoma (Cangzhu)

$\rightarrow$ Zäher, gelber Schleim, thorakaler Druck: + Trichosanthis semen (Gualouren), Fritillariae thunbergii bulbus (Zhebeimu), alternativ „Kleines Dekokt gegen Vernichtungsgefühl in der Brust“" (Xiao xianxiong tang)

\subsubsection{Calor ("Hitze", re) und Stagnation von Toxischem blockieren den 0 . pulmonalis ( $\mathrm{Fk}_{\text {"Lunge", }} \mathrm{fei}$ )}

- Fortgeschrittenes Krankheitsstadium mit zunehmender Atemnot, erschwerter und beschleunigter Atmung

- Symptome

$\rightarrow$ Schwere Atemnot, beschleunigte flache Atmung, Cyanose

$\rightarrow$ Husten ohne oder mit wenig zähem gelblichem Schleim, Druck- bzw. Engegefühl

$\rightarrow$ Fieber, Hitzegefühl, Schwäche, Schweregefühl, stark reduzierter Allgemeinzustand

$\rightarrow$ Evtl. Unwohlsein, Appetitlosigkeit, Obstipation, dunkler, spärlicher Urin

$\rightarrow$ Zunge: stark gerötet, evtl. livide, Belag trocken, dick, gelb bzw. gelbbraun

$\rightarrow$ Pulse: lubrici („schlüpfrig“, hua), evtl. celeri („beschleunigt“, shu)

- Therapieprinzip

$\rightarrow$ Erweichen und Umwandeln von pituita („Schleim“, tan)

$\rightarrow$ Umwandeln und Kanalisieren von humor („Feuchtigkeit“, shi) bzw. pituita („Schleim“, tan)

$\rightarrow$ Klären und Ableiten von calor („Hitze“, re) und Toxischem

$\rightarrow$ Entfalten des o.pulmonalis (Fk „Lunge“, fei)

- Covid-19 C: Rezeptur zum Eliminieren von calor („Hitze“, re), humor („Feuchtigkeit", shi) und Stagnation von Toxischem zur Entfaltung des o. pulmonalis (Fk "Lunge“, fei), (siehe Kasten links)

- Modifikationen

$\rightarrow$ Trockenheit, zäher Schleim: + Phragmitis rhizoma (Lugen), Trichosanthis radix (Tianhuafen), Fritillariae cirrhosae bulbus (Chuanbeimu) 
$\rightarrow$ Feuchter Zungenbelag: + Coicis semen (Yiyiren), Benincasae semen (Dongguazi)

$\rightarrow$ Ableiten von calor („Hitze“, re): + Achyranthis bidentatae radix (Niuxi)

$\rightarrow$ Klären von calor („Hitze“, re): + Anemarrhenae rhizoma (Zhimu), Phragmitis rhizoma (Lugen)

$\rightarrow$ Ausgeprägte Cyanose, livide Zunge: + Curcumae longae tuber (Yujin), Notoginseng radix (Sanqi)

Dieses Stadium dürfte wie schon erwähnt das letzte sein, das TCM-Therapeuten selbst im günstigsten Fall zugänglich sein wird. Darum ergänze ich meinen Essay lediglich noch um Vorschläge zur Nachbehandlung nach überstandener Akutphase.

\subsection{Nachbehandlung nach überstandener Akutphase einer Covid- 19-Erkrankung}

In dieser Phase leiden viele Patienten, auch wenn sie keine schwere Pneumonie durchgemacht haben, unter den Folgen der massiven Virusreplikation in der Lunge und der konsekutiven heftigen Immunreaktion. Die Erkrankung selbst und auch maschinelle Beatmung schwächen das Qi, nach einer schweren Pneumonie mit calor-Aspekten („Hitze“, re) und Toxischem ist das Yin dezimiert, letztlich ist auch die Transformation und der Transport der Flüssigkeiten oft nachhaltig gestört. Die Auswahl der Konzepte wird auch in der Rekonvaleszenz nach Zeichen und Klinik erfolgen.

Mögliche Grundrezepturen:

- Qi-Defizit des o. pulmonalis (Fk „Lunge“, fei): $\rightarrow$ „Dekokt zur Stützung des Fk Lunge (o. pulmonalis)“"(Bufei tang)

- Ergänzung des Qi und Stabilisierung der extima (Oberfläche, biao): $\rightarrow$ „Pulver gegen Wind aus Jade" (Yu pingfeng san)

- Trockenheit und Yin-Defizit des o. pulmonalis (Fk „Lunge“, fei): $\rightarrow$ „Dekokt zur Festigung des Metalls mit Lilium“ (Baihe gujin tang), $\rightarrow$ „Glehnia- und Ophiopogon-Dekokt" (Shashen maidong tang)

- Trockenheit mit eingetrockneter pituita („Schleim“, tan): $\rightarrow$ „Pulver mit Fritillaria und Trichosanthes" (Beimu gualou san)

\section{Schlussbemerkungen}

Nachdem sich die Behandlung mit chinesischen Arzneimitteln bereits während des SARS-Ausbruchs 2003 als effektiv erwiesen hat, gibt es auch in der aktuellen SARS-CoV-2-Pandemie entsprechende Therapiekonzepte, die in China erfolgreich angewendet worden sind. Die zentrale und primär zu behandelnde Pathologie ist gekennzeichnet durch epidemisches humor-Toxisches („Feuchtigkeit“, shi), das den o. pulmonalis (Fk „Lunge“, fei) affiziert und zur Blockade des Energieflusses und der Qi-Transformation führt. Im weiteren Verlauf der Erkrankung kann es sekundär zu calor-Befunden („Hitze“, re) und Stagnation von Toxischem kommen, die mit den vorgestellten Konzepten - orientiert an klinischen Zeichen und Stadien der Erkrankung - positiv beeinflusst werden können.

\section{Quellen und Links}

1. „SARS - Clinical trials on treatment using a combination of Traditional Chinese medicine and Western medicine; Report of the WHO International Expert Meeting to review and analyse clinical reports on combination treatment for
In Modifikationen verwendbare Arzneimittel

Klassische Rezepturen für die Rekonvaleszenz 
SARS“, 8-10 October 2003, Beijing, People’s Republic of China, World Health Organization, Geneva 2004.

2. „How Coronavirus (COVID-19) is treated with TCM in China“, John Chen, Pharm.D., Ph.D., O.M.D., L.Ac., www.elotus.org.

3. Nachfolgend die Webadressen, deren Übersetzung Axel Wiebrecht zur Verfügung gestellt hat und auf denen obige Ausarbeitung beruht: http://www.nhc.gov. $\mathrm{cn} / \mathrm{xcs} /$ zhengcwj/202003/46c9294a7dfe4cef80dc7f5912eb1989/files/ ce3e6945832a438eaae415350a8ce964.pdf, Generalbüro der Nationalen Hygieneund Gesundheitskommission und das Büro der staatlichen Verwaltung für Traditionelle Chinesische Medizin, abgerufen am 4.3.2020; http://m.china.caixin. $\mathrm{com} / \mathrm{m} / 2020-03-04 / 101523776 . \mathrm{html}$ ? $\mathrm{cxw}=\mathrm{IOS} \& S$ from $=$ Wechat\&originReferrer $=\mathrm{iOS}$ share\&from $=$ singlemessage\&isappinstalled $=0$, abgerufen am 4.3.2020.

4. „Medical Records from a Young and Brave Female Traditional Chinese Medicine (TCM) doctor on Fighting the COVID-19“, Chen Juan, Huang Di, Wang Shi Qi, Cai Xiang, zusammengestellt und ins Englische übersetzt von John K. Chen, Pharm.D., PhD., OMD, Lac.

5. „Initial Thoughts on Coronavirus Prevention and Treatment with Chinese Medicine“; Heiner Fruehauf, classicalchinesemedicine.org.

6. „Report from the Front Line in Wuhan“; Liu Lihong, übersetzt von Heiner Fruehauf, classicalchinesemedicine.org.

7. „The Dampness Epidemic: Exploring the Clinical Characteristics of COVID-19 in Shanghai"; Xue Yan, Zhang Wei, Xu Guihua, Chen Xiaorong, Lu Yunfei, Wang Zhenwei, Shi Kehua, Wu Huan, Yu Jian, Shanghai Journal of Traditional Chinese Medicine and Pharmacology (Shanghai zhongyiyao zazhi), 5. März 2020, bearbeitet und übersetzt von Heiner Fruehauf, National University of Natural Medicine, College of Classical Chinese Medicine.

8. https://www.tcm.edu/news/detail/news/corona-covid-19-und-therapiemoeglichkeiten-der-chinesischen-medizin/

9. „Approaching covid-19 as damp toxin“; Jin Zhao, Chris Flanagan, The Lantern Vol: 17-2a, März 2020.

\section{Zum Autor}

Dr. med. Christian Thede (geb. 1960), Arzt für Allgemeinmedizin, Praxis für Chinesische Medizin seit 1993, Dozent für Chinesische Medizin bei der SMS, ehemaliger Dozent der Ärztekammer Hamburg sowie ehemaliger Lehrbeauftragter an der Universität Witten-Herdecke, Mitglied im Prüfungsausschuss Akupunktur der Ärztekammer Schleswig-Holstein, Mitautor des Buches Leitfaden Chinesische Rezepturen.

Korrespondenzanschrift:

Dr. med. Christian Thede

Gorch-Fock-Weg 16

23730 Neustadt/Holstein

E-Mail:praxis@christianthede.de

Einhaltung ethischer Richtlinien

Interessenkonflikt: Christian Thede gibt an, dass kein Interessenkonflikt besteht. Dieser Beitrag beinhaltet keine Studien an Menschen oder Tieren. 